\title{
Effects of lipid peroxide formation in fowl semen on sperm motility, ATP content and fertilizing ability
}

\author{
G. J. Wishart \\ Agricultural Research Council's Poultry Research Centre, Roslin, Midlothian EH25 9PS, U.K.
}

\begin{abstract}
Summary. The ability of samples of semen from individual male fowl to form the products of lipid peroxidation during $5 \mathrm{~h}$ aerobic incubation at $40^{\circ} \mathrm{C}$ varied between 0 and $8 \mathrm{nmol}$ malonaldehyde $/ 10^{9}$ spermatozoa. Formation of higher concentrations of malonaldehyde was associated with a partial or complete loss of fertilizing ability whilst the fertilizing ability of samples producing low or negligible concentrations of malonaldehyde remained unimpaired. The semen of birds which showed a tendency to form high concentrations of malonaldehyde was not readily identifiable as abnormal by assessment of sperm motility, morphology or ATP content. Nor was the loss of fertilizing ability during aerobic incubation associated with an obvious change in these characteristics.
\end{abstract}

\section{Introduction}

An oxygenated environment was shown to be necessary for the optimal metabolism of fowl and turkey spermatozoa when incubated at physiological temperatures in vitro (Wishart, 1982). Oxygenated conditions also improved the maintenance of the fertilizing capacity of fowl and turkey semen when held at $5-10^{\circ} \mathrm{C}$ (Wishart, 1981; Lake, Cherms \& Wishart, 1984), the conventional temperature range for practical semen storage (see Lake \& Stewart, 1978). Conversely for improving the storage of mammalian semen at such low temperatures, efforts have been made to exclude oxygen from the sperm environment (Mann \& Lutwak-Mann, 1981), because byproducts of the normal oxidative metabolism of these spermatozoa-free radicals of oxygen and $\mathrm{H}_{2} \mathrm{O}_{2}$-induce the formation of lipid peroxides which are extremely toxic to the spermatozoa (see Mann \& Lutwak-Mann, 1981).

Since there is current interest in the aeration of poultry semen during storage, the present work sought to investigate whether incubated fowl semen would produce lipid peroxides and the effect of any formed peroxides on the viability and fertilizing ability of fowl spermatozoa.

\section{Materials and Methods}

Birds and semen treatment. Male fowl were from a layer-type control strain obtained from Ross Poultry Ltd, Newbridge, Midlothian and hens were of a commercial layer strain. Birds were caged individually, given $14 \mathrm{~h}$ light/ $24 \mathrm{~h}$ and fed a commercial breeder's ration ad libitum. Semen was collected from the males on a twice-weekly routine.

Numbers of spermatozoa were estimated using a Coulter Counter or by relating DNA content (Burton, 1956) of samples to numbers of spermatozoa. Semen was diluted 4-fold in a glutamatebased buffer, $\mathrm{pH} 7 \cdot 4$ (Wishart, 1982) containing, per ml, 100 units penicillin, $100 \mu \mathrm{g}$ streptomycin 
and $10 \mu \mathrm{g}$ kanamycin. Samples were incubated aerobically in a shaking water bath at $40^{\circ} \mathrm{C}$ in $25 \mathrm{ml}$ 'Nalgene' flasks (2-3 ml diluted semen) or $18 \mathrm{~mm}$ diameter polycarbonate vials $(0.6 \mathrm{ml}$ diluted semen). For anaerobic incubations samples of $1 \mathrm{ml}$ were held under liquid paraffin in $10 \mathrm{~mm}$ diameter polycarbonate tubes.

Assays. The products of lipid peroxidation were assayed as malonaldehyde by the thiobarbituric acid reaction (Barber \& Bernheim, 1967). The measurement of malonaldehyde has been shown to be representative of the presence of lipid peroxides, of which it is a breakdown product (see Alvarez \& Storey, 1982). In this text 'lipid peroxides' will be used synonymously with malonaldehyde.

Sperm ATP concentrations were assayed using firefly luciferase (Strehler, 1974) as described previously (Wishart, 1981).

Sperm motility was measured by a modification of the method of Atherton, Cisson, Wilson \& Golder (1980). Incubation mixtures of spermatozoa, at $1: 4$ dilution of semen, were further diluted 50 -fold in $\mathrm{Ca}^{2+}$ - and $\mathrm{Mg}^{2+}$-free phosphate buffered saline (Dulbecco \& Vogt, 1954) at $30^{\circ} \mathrm{C}$ and pumped through a $10-\mathrm{mm}$ flow cell (regulated at $30^{\circ} \mathrm{C}$ ) in a Unicam SP 500 spectrophotometer. The reduction in optical density at $550 \mathrm{~nm}$ after stopping the flow of medium, as a percentage of the initial optical density, was taken as an estimate of sperm motility.

Sperm morphology and eosin uptake were assessed microscopically in nigrosin-eosin smears as described by Lake \& Stewart (1978).

Fertility testing. The fertilizing ability of semen samples was assessed by inseminating $0.12 \mathrm{ml}$ of a 4-fold dilution of semen, containing approximately $150 \times 10^{6}$ spermatozoa, intravaginally into hens. The percentage of fertile eggs in a sample of 56-69 eggs laid by groups of 8 hens during Days 2-11 after insemination was calculated. Evidence of fertilization was determined by visual examination of embryos between Days 4 and 8 of incubation.

To obtain sufficient semen for fertility trials, samples from the pairs of birds within each of the 2 groups identified in Table 1 were further paired to form 3 high-activity and 3 low-activity malonaldehyde-producing samples, each sample consisting of semen from 4 birds. These samples were then tested for fertilizing ability and malonaldehyde production before and after aerobic incubation for $5 \mathrm{~h}$ at $40^{\circ} \mathrm{C}$ on two separate occasions. Thus Text-fig. 2 shows 6 experiments using samples from the high activity group (Samples 2-7) and a composite (Sample 1) of 6 experiments using semen from the low activity groups.

\section{Results}

\section{Malonaldehyde formation by fowl semen}

Preliminary tests on the formation of lipid peroxides by aerobically-stored samples of semen, pooled periodically from randomly-chosen male fowls, yielded variable results. To investigate the contribution of semen from different males to this variability, the 48 available birds were grouped as 24 pairs and peroxide formation in the semen of each pair was assessed on 5 separate occasions during a 5-h aerobic incubation at $40^{\circ} \mathrm{C}$. From the 24 samples of semen, two groups of 6 were identified: one in which samples produced $>1.3 \mathrm{nmol}$ malonaldehyde $/ 10^{9}$ spermatozoa on each occasion, and one in which samples consistently produced $<0.25 \mathrm{nmol}$ malonaldehyde $/ 10^{9}$ spermatozoa (Table 1). The remaining 12 samples of semen largely resembled the latter group, but occasionally produced levels of malonaldehyde $>0.25$ but not exceeding $0.75 \mathrm{nmol}$ malonaldehyde $/ 10^{9}$ spermatozoa.

During aerobic incubation for $5 \mathrm{~h}$, the formation of malonaldehyde proceeded linearly to levels of $5 \mathrm{nmol} / 10^{9}$ spermatozoa (Text-fig. 1). However, an aliquant of the same semen sample incubated under anaerobic conditions produced, at $0.4 \mathrm{nmol} / 10^{9}$ spermatozoa, barely significant levels of malonaldehyde (Text-fig. 1). 


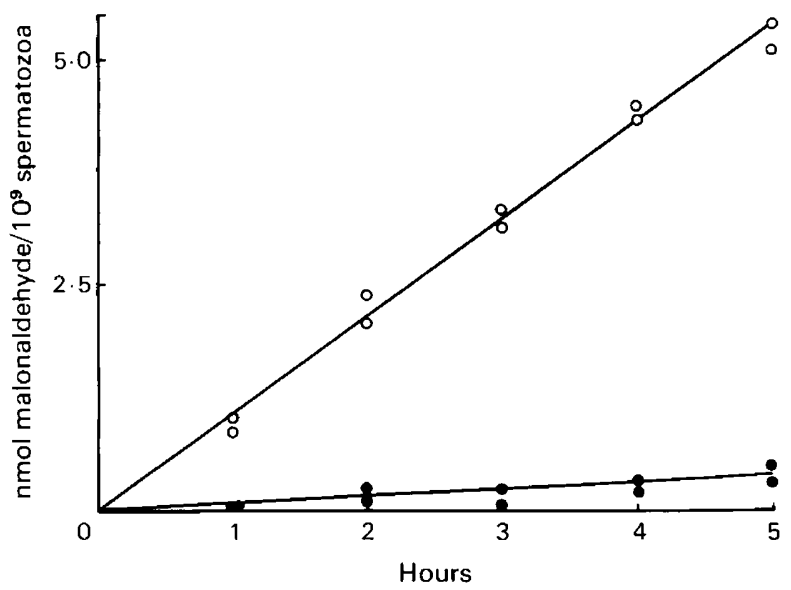

Text-fig. 1. Rate of production of lipid peroxides by fowl semen in vitro at $40^{\circ} \mathrm{C}$. Each point represents one determination on a semen sample pooled from 6 birds and incubated as 4 diluted aliquants -2 aerobically $(O)$ and 2 anaerobically $(O)$. Linear regressions \pm standard error were $1.065 \pm 0.015$ (aerobic sample) and $0.062 \pm 0.009$ (anaerobic sample) $\mathrm{nmol}$ malonaldehyde $/ 10^{9}$ spermatozoa $/ \mathrm{h}$.

Formation of high concentrations of malonaldehyde required the presence of spermatozoa. Samples of semen diluted $1: 4 \mathrm{in}$ buffer and incubated aerobically for $5 \mathrm{~h}$ at $40^{\circ} \mathrm{C}$ produced $4.54 \pm$ $0.69 \mathrm{nmol}$ malonaldehyde $/ \mathrm{ml}$ whilst the same samples incubated after prior centrifugation at $800 \mathrm{~g}$ for $15 \mathrm{~min}$, to remove spermatozoa, produced $0.74 \pm 0.27 \mathrm{nmol}$ malonaldehyde $/ 5 \mathrm{ml}$ (values mean \pm s.e.m. of 4 different samples).

Contamination of semen samples did not appear to be a factor in determining the degree of formation of peroxides. Although samples with a high rate of peroxide formation were apparently free of contamination, it was considered difficult to be absolutely certain because of the method of semen collection and the anatomy of the avian cloaca (see Lake, 1981). However, it was ascertained that the normally low rates of lipid peroxide formation by certain samples were unaffected by deliberate contamination of semen with urates or 'transparent fluid'.

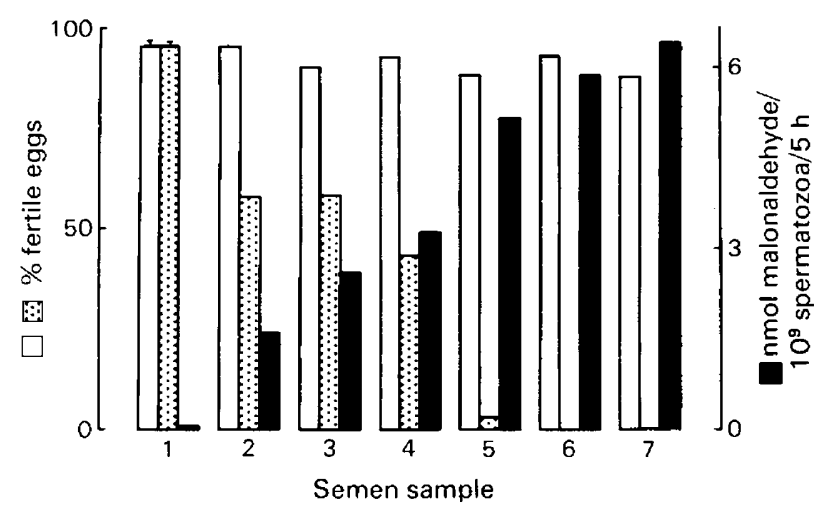

Text-fig. 2. Effect of production of lipid peroxides on the fertilizing ability of incubated semen samples. Fertilizing ability is shown before ( $\square$ ) and after (O) a 5 -h incubation at $40^{\circ} \mathrm{C}$. Peroxides produced during this incubation $(\square)$ are shown for each experiment. Experiment 1 represents a composite (mean \pm s.e.m.) of 6 separate experiments using semen from the low peroxide-producing group (see 'Materials and Methods' for details). All experiments used semen pooled from 4 males. 
Malonaldehyde formation and sperm viability

The formation of lipid peroxides (Table 1; Text-fig. 1) and their effect on the fertilizing ability of fowl spermatozoa (Text-fig. 2) were studied in pooled semen samples. To investigate further the features of samples with a high rate of peroxide formation, the semen from individual males was examined.

Of the 12 male fowls contributing semen to the 6 highly active samples of Table 1 , it was found that 6 birds, one from each pair, were producing semen which was largely responsible for the high levels of lipid peroxides (Birds 7-12 of Text-fig. 3). Production of lipid peroxides by samples from these birds and from 6 individuals from the 'low peroxide' forming group of Table 1 are shown in Text-fig. $3 \mathrm{a}$ in comparison with sperm motility (Text-fig. 3b), ATP content (Text-fig. 3c) and morphology (Text-fig. 3d).

Table 1. Lipid peroxide production by semen samples taken from 12 pairs of male fowl and displaying different levels of activity

\begin{tabular}{cc}
\hline Low activity group & High activity group \\
\hline 0 & $1.76 \pm 0.15$ \\
$0.13 \pm 0.05$ & $2 \cdot 33 \pm 0.17$ \\
$0.03 \pm 0.03$ & $2 \cdot 51 \pm 0.46$ \\
0 & $2.37 \pm 0.21$ \\
$0.04 \pm 0.02$ & $4.55 \pm 0.96$ \\
0 & $7.08 \pm 0.64$ \\
\hline
\end{tabular}

Each value, as nmol malonaldehyde produced/ $10^{9}$ spermatozoa during aerobic incubation for $5 \mathrm{~h}$ at $40^{\circ} \mathrm{C}$, represents mean \pm s.e.m. of 5 experiments performed on different days using semen from the same pairs of males.

(a) Lipid peroxide production

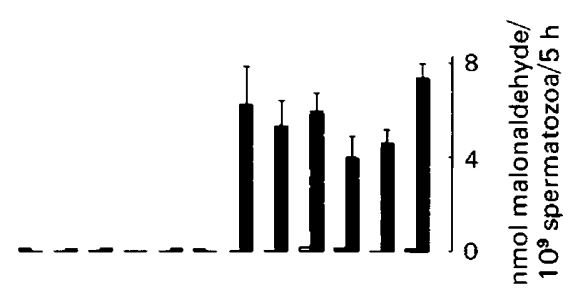

(c) Sperm ATP conc.

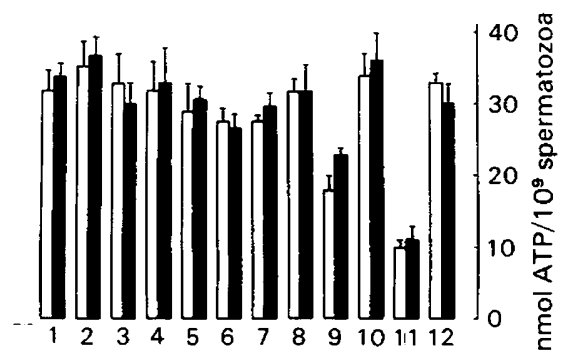

(b) Sperm motility

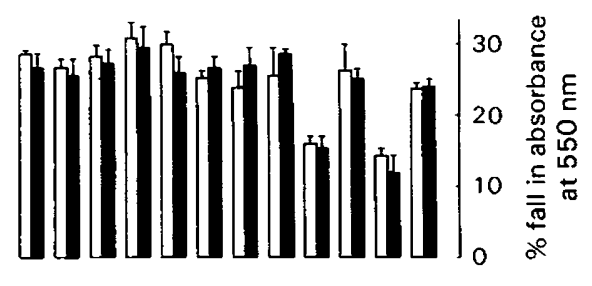

(d) Sperm morphology

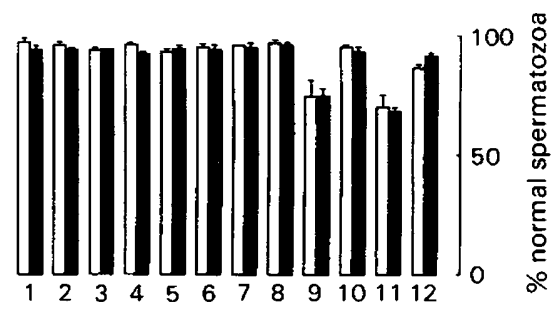

Semen donor

Text-fig. 3. Comparison of lipid peroxide production by fowl semen during aerobic incubation for $5 \mathrm{~h}$ at $40^{\circ} \mathrm{C}$ with sperm motility, morphology and ATP content. Results (mean \pm s.e.m. from 3 separate experiments) are shown before $(\square)$ and after $(\square)$ incubation. See text for details. 
The concentrations of spermatozoa in semen samples from Birds $7-12$ were: $5 \cdot 12 \pm 0.41,5.97$ $\pm 0.55,5.71 \pm 0.25,4.27 \pm 0.17,4.60 \pm 0.44$ and $7.67 \pm 0.09 \times 10^{9}$ spermatozoa $/ \mathrm{ml}$ semen, respectively (mean \pm s.e.m. of 3 separate samples from each bird). With one exception, these were not significantly lower than $5.12 \pm 0.15 \times 10^{9}$ spermatozoa $/ \mathrm{ml}$ semen, this being the mean \pm s.e.m. of 3 separate samples from each of Birds 1-6. The fertilizing abilities of fresh semen samples from Birds 7-12, respectively, were demonstrated by the fact that $92,91,90,91,96$ and $94 \%$ fertile eggs were laid by groups of 8 hens during the 10 days after insemination.

\section{Discussion}

The formation of the products of lipid peroxidation by fowl spermatozoa has been noted on one previous occasion (Fujihara \& Howarth, 1978). The amounts produced by washed spermatozoa were reported to be only $10 \%$ of that in the more active samples described in the present work. This may be partly due to differences in the semen donors, but perhaps also to the incubation conditions used by Fujihara \& Howarth (1978), i.e. diluted semen stored in tubes. Under certain conditions such samples may become anaerobic (Wishart, 1981). Furthermore, preliminary work (G. J. Wishart, unpublished result) shows that washed spermatozoa, even from the low-activity samples (see 'Results'), produce large amounts of lipid peroxides when deprived of seminal plasma.

The present work shows that samples of diluted semen from certain individual birds ( 6 out of 48 studied) produce lipid peroxides at a rate which is 70 -fold greater than that of samples from another group with a low rate of peroxide production (see Text-fig. 3a). The high levels of peroxides were produced by samples from these individual birds alone (Text-fig. 3a) or when mixed with samples of semen with a low rate of lipid peroxide production (Table $1 \&$ Text-fig. 2).

At levels of formed lipid peroxides which severely inhibited the fertilizing ability of fowl semen samples (Text-fig. 2), sperm motility, ATP content and morphology remained unaffected (Text-fig. 3). This is contrary to the situation with mammalian spermatozoa for which a loss of motility was correlated with the formation of lipid peroxides by ram (Jones \& Mann, 1976) and rabbit (Alvarez \& Storey, 1982) spermatozoa. Furthermore, damage to ram spermatozoa, apparently by peroxidation of endogenous phospholipids, was reported to include enzyme leakage, morphological damage and loss of intracellular ATP (Jones \& Mann, 1977). These differences would not appear to be the result of a greater rate of formation of lipid peroxides by mammalian spermatozoa since rabbit spermatozoa form lipid peroxides (Alvarez \& Storey, 1982) at the same rate as did spermatozoa from Birds 7-12 (Text-fig. 3a), with concomitant loss of motility. It may be that mammalian spermatozoa are more sensitive to the adverse effects of lipid peroxides and that more prolonged incubation of fowl semen in the presence of high levels of lipid peroxides may lead to the eventual loss of various characteristics such as sperm motility and ATP content. However, from the present work the critical effect of lipid peroxide formation that leads to the loss of fertilizing ability of fowl spermatozoa remains unidentified.

The semen samples that had the potential to produce high levels of lipid peroxides were not readily identifiable as abnormal by examination of fresh semen. Only 2 out of 6 samples studied in Text-fig. 3 had semen with low sperm motility, ATP content and morphological integrity; only one of these showed an abnormally low concentration of spermatozoa; and all 6 samples, when inseminated immediately after collection, fertilized $90 \%$ or more of eggs laid by groups of hens. Furthermore, these same semen samples, which produced high levels of lipid peroxides when stored in vitro, showed little thiobarbituric acid-reactive material when freshly collected. Therefore the formation of lipid peroxides in vivo appears to be inhibited.

Since the actual mechanism of lipid peroxide formation by fowl spermatozoa remains unidentified, it is not yet known whether the presence or absence of some factor leads to the production of high levels of lipid peroxides. Clearly the identification and control of such factor(s) could improve the storage of aerated poultry semen. 
I thank Mrs F. Ross for skilled assistance and Dr P. E. Lake for helpful discussion.

\section{References}

Alvarez, J.G. \& Storey, B.T. (1982) Spontaneous lipid peroxidation in rabbit epididymal spermatozoa: its effect on sperm motility. Biol. Reprod. 27, 1102-1108.

Atherton, R.W., Cisson, C.M., Wilson, B.M. \& Golder, T.K. (1980) Quantitation of avian spermatozoan motility: neurochemical regulation. Gamete Res. 3, 17-24.

Barber, A.A. \& Bernheim, F. (1967) Lipid peroxidation: its measurement, occurrence and significance in animal tissues. Adv. Geront. Res. 2, 355-403.

Burton, K. (1956) A study of the conditions and mechanisms of the diphenylamine reaction for the calorimetric estimation of deoxyribonucleic acid. Biochem. J. 62, 315-323.

Dulbecco, R. \& Vogt, M. (1954) Plaque formation and isolation of pure lines with poliomyelitis viruses. $J$. exp. Med. 99, 167-199.

Fujihara, N. \& Howarth, B. (1978) Lipid peroxidation in fowl spermatozoa. Poult. Sci. 57, 1766-1768.

Jones, R. \& Mann, T. (1976) Lipid peroxides in spermatozoa: formation, role of plasmalagen and physiological significance. Proc. R. Soc. B 193, 317333.

Jones, R. \& Mann, T. (1977) Damage to ram spermatozoa by peroxidation of endogenous phospholipids. $J$. Reprod. Fert. 50, 261-268.
Lake, P.E. (1981) Male genital organs. In Form and Function in Birds, Vol. 2, pp. 1-62. Eds A. S. King \& J. McLelland. Academic Press, London.

Lake, P.E. \& Stewart, J.M. (1978) Artificial insemination in poultry. Bull. Min. Ag., Fish. Food, No. 213. HMSO, London.

Lake, P.E., Cherms, F.L. \& Wishart, G.J. (1984) Effect of aeration on the fertility of turkey semen stored for 48 $h$ at $5^{\circ}$ and $15^{\circ} \mathrm{C}$ : a study over a 14-week breeding period. Reprod. Nutr. Développ. (in press).

Mann, T. \& Lutwak-Mann, C. (1981) Male Reproductive Function and Semen, pp. 212-217. Springer Verlag, New York.

Strehler, B.J. (1974) Adenosine-5'-triphosphate and creatine phosphate. Determination with luciferase. In Methods of Enzymic Analysis, pp. 2112-2116. Ed. H. U. Bergmeyer. Academic Press, New York.

Wishart, G.J. (1981) The effect of continuous aeration on the fertility of fowl and turkey semen stored above $0^{\circ}$ C. Br. Poult. Sci. 22, 445-450.

Wishart, G.J. (1982) Maintenance of ATP concentrations in and of fertilizing ability of fowl and turkey spermatozoa in vitro. J. Reprod. Fert. 66, 457-462.

Received 18 August 1983 